\title{
Stress-Induced Twin Boundary Motion in Particulate Ni-Mn-Ga /Polymer Composites
}

\author{
J. Feuchtwanger ${ }^{1, a}$, M. L. Richard ${ }^{2, b}$, P. Lázpita ${ }^{1, c}$, J. Gutierrez ${ }^{1, d}$, \\ J. M. Barandiaran ${ }^{1, \mathrm{e}}$, S. M. Allen ${ }^{3, \mathrm{f}}$ and R. C. O'Handley ${ }^{3, \mathrm{~g}}$ \\ ${ }^{1}$ Universidad del País Vasco, Depto. Electricidad y Electrónica, Barrio de Sarriena S/N, Leioa, \\ Vizcaya 48940, Spain \\ ${ }^{2}$ The Richard Stockton College of NJ, Department of Chemistry, PO Box 195, Pomona, NJ 08240 \\ USA \\ ${ }^{3}$ Massachusetts Institute of Technology, Department of Materials Science, 77 Massachusetts \\ Ave., Cambridge, MA 02139, USA \\ a bcpfemoj@ehu.es, ${ }^{b}$ marc.richard@stockton.edu, ${ }^{c}$ weblaarp@lg.ehu.es, ${ }^{d}$ jon@we.lc.ehu.es, \\ emanub@we.lc.ehu.es, ${ }^{f}$ smallen@mit.edu, ${ }^{9}$ bobohand@mit.edu
}

Keywords: Ferromagnetic Shape Memory Alloys, Composites, Mechanical Energy Absorption, Xray Diffraction, Neutron Diffraction.

\begin{abstract}
Composites of $\mathrm{Ni}-\mathrm{Mn}-\mathrm{Ga}$ particles in a polyurethane matrix can be made by mixing the particles with the polymer, and allowing them to cure under a magnetic field to texture the composites. These composites show large hysteresis and mechanical losses, when subjected to a cyclic stress, that were far larger than the matrix polymer ones. The additional losses are attributed to the motion of twin boundaries in the filler particles and provide a way for obtaining mechanical energy absorption in a wide frequency range. By means of X-ray and neutron diffraction we present evidence that confirms that twins are present in the particles and that they do move when mechanically loading the composite
\end{abstract}

\section{Introduction}

Ferromagnetic shape memory alloys (FSMAs) are a kind of active materials that exhibit large plastic deformations due to mechanical stress or magnetic field induced twin boundary motion. They are attractive because they combine the large strains associated with conventional shape memory alloys (SMAs) and bandwidth approaching that of magnetostrictive or piezoelectric materials. For off-stoichiometric, tetragonal NiMnGa a $6 \%$ strain has commonly been reported in the literature [1] and strains of up to $10 \%$ have been reported in the orthorhombic phase [2]. These strains are comparable to the strains achieved in NiTi, one of the most widely used SMAs [3]. Continuous cyclic actuation of FSMA actuators has been demonstrated up to $800 \mathrm{~Hz}$, this frequency was limited by the inability of the power supply used to drive the magnetic field required for actuation at those frequencies, rather than by material limitations [4]. Marioni showed that the material itself can respond at an equivalent frequency of about $1.5 \mathrm{kHz}$ [5].

However, FSMAs also share one of the less desirable traits of the SMAs, they can exhibit a large hysteresis in their stress-strain loops. While the large hysteresis degrades the performance of the material as an actuator, it makes these alloys very attractive for damping applications. The large loss in these materials is associated with the anelastic motion of twin boundaries and the resulting pseudoplastic deformation of the material, that can be reversed by applying a stress in the direction opposite to the stress that originally deformed the material, or by applying a stress normal to the previous one. Plastic deformation has been studied as a damping mechanism because it can 
dissipate large amounts of energy and the loss is largely independent of frequency. However plastic deformation is not practical for cyclic damping, because the amount of energy dissipated decays after each consecutive cycle [6]. The pseudo-plastic deformation seen in FSMAs shows the large energy absorption per cycle of plastic deformation, but unlike plastic deformation, its magnitude does not appear to decrease with the number of stress cycles.

The loss per unit volume per cycle for a single crystal FSMA can be approximated by the product of the threshold stress for twin boundary motion and the twining strain. Using typical values for $\mathrm{Ni}-$ $\mathrm{Mn}-\mathrm{Ga}$ alloys, this product is of the order $2 \mathrm{MPa} \times 6 \%$ or $120 \mathrm{~kJ} / \mathrm{m}^{3}$. This can be compared to the energy loss per cycle in a polymer $(\Delta)$, that can be obtained by $\Delta=2 \pi U \tan (\delta)$

where $U$ is the total energy input per cycle, and $\delta$ is the phase lag between the applied stress and the resulting strain in the polymer. The total energy per cycle is given by $U=1 / 2 E \varepsilon^{2}$, where

$E$ is the modulus of the material and $\varepsilon$ is the maximum strain. Typical values for $E$ and $\tan (\delta)$ can be obtained from the stiffness- loss map in Wang [7]. For a rubbery matrix typical values of $E \approx 1$ $\mathrm{MPa}$ and $\tan (\delta) \approx 0.5$ respectively. For a maximum deformation on the polymer equal to the twinning strain in the FSMA $(\varepsilon=0.06)$, the loss per cycle is approximately $15 \mathrm{~kJ} / \mathrm{m}^{3}$. From this comparison it can be seen that FSMA's have the ability to dissipate almost an order of magnitude more of energy that a polymer alone would dissipate under similar conditions.

The use of single crystals of $\mathrm{Ni}-\mathrm{Mn}-\mathrm{Ga}$ for damping applications poses several challenges; the single crystals are very brittle and do not stand up well to tensile loads. Single crystals are expensive relative to conventional visco-elastic materials, like rubber. The use of composites made from an FSMA filler in a visco-elastic matrix ameliorates most of these issues while still exhibiting a marked improvement in the energy dissipation capability when compared to the matrix material. $\mathrm{Ni}-\mathrm{Mn}-\mathrm{Ga}$ has a high magneto-crystalline anisotropy, because of that it is possible to have the particles oriented in the composite if a magnetic field is applied to the FSMA/ polymer slurry while the matrix cures. If the particles used in making the composites are mostly single crystals and they also are single variant, the unique axis ( $c$-axis) of the martensitic particles can be aligned with the direction of the magnetic field applied while curing. A similar approach has been successfully applied to Terfenol-D, a commonly used magneto-strictive material [8], using the same technique $[9,10]$, to overcome similar issues. Unlike $\mathrm{Ni}-\mathrm{Mn}-\mathrm{Ga}$, Terfenol-D has a low magneto-crystalline anisotropy, so the needle-like particles with a $\langle 112\rangle$ direction along the long axis are used. In this case the shape anisotropy is used to obtain the fiber texture in the composite, with the easy axis along the direction of the field used during curing, instead of the magneto-crystalline anisotropy. In $\mathrm{Ni}-\mathrm{Mn}-\mathrm{Ga}$ the high magneto-crystalline anisotropy $\left(\sim 1.5 \times 10^{5} \mathrm{~J} / \mathrm{m}^{3}\right)$ allows for the composites to be textures without the need for particles with a long dimension along the $\langle 001\rangle$ direction.

The use of magnetic measurements, electron microscope micrographs, and diffraction experiments will be used to show the ability of twin boundaries to move under an applied load. Similar results have been published by Scheerbaum et al. [11] Hinz et al. [12], Gans et al. [13], as well as by the authors $[14,15,16,17,18]$.

\section{Experimental}

For the experiments described in this paper composites were made from spherical particles of NiMn-Ga ranging in size from $25-75 \mu \mathrm{m}$ made by spark erosion at the University of California San Diego as described in $[19,20]$, and a two part, zero volume change, polyurethane made by Lord Adhesives. The mass of the FSMA and the polyurethane were chosen such that the resulting composites would have a $25 \%$ by volume fraction of FSMA. In all cases polytetrafluoroethylene molds were used, and a $4 \mathrm{kOe}$ field was applied while the polymer cured. For the mechanical tests samples were cast into I beam shapes, with the top and bottom sections comprised of aluminum endcaps with a dovetail shaped opening that lead into a straight section. The central part of the sample was $10 \mathrm{~mm}$ long and had a uniform cross-section of $5 \mathrm{~mm} \times 5 \mathrm{~mm}$. The aluminum endcaps were used to grip the sample and avoid the sample from slipping out of the grips. The magnetic field was applied parallel to the long dimension of the sample, a schematic of the can be seen in Fig. 
1 a). For all other tests samples where cast into sheets $1 \mathrm{~mm}$ thick and $10 \mathrm{~mm} \times 15 \mathrm{~mm}$. These samples were made with the magnetic field applied in two different orientations relative to the sheet; for the first kind the magnetic field was applied along the direction normal to the sheet plane (Fig. $1 \mathrm{~b}$ )), for the second kind the field was applied in the plane of the sample along the long direction of the sample (Fig. $1 \mathrm{c})$ ).
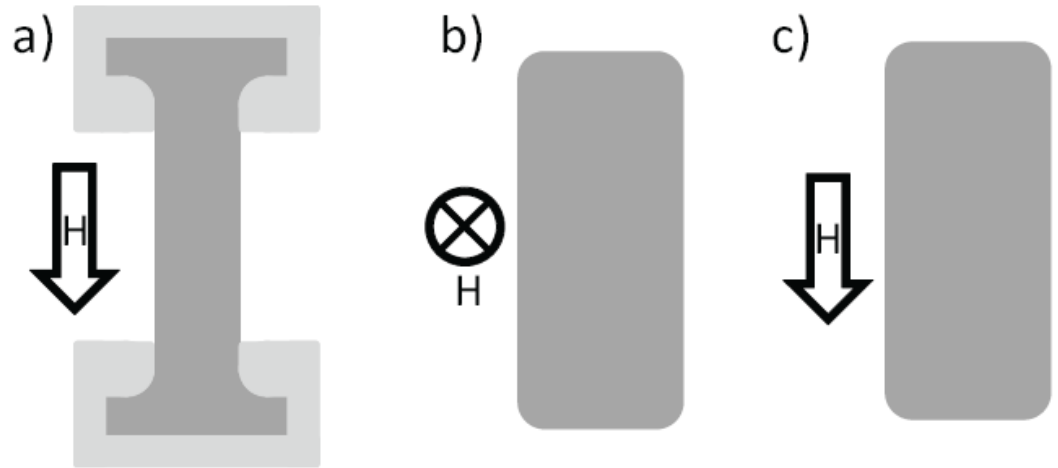

Figure 1 Schematic of the different sample geometries used in the experiments. The samples for mechanical testing are depicted in a), the lighter gray represents the Aluminum parts and the darker gray the FSMA/polymer composite. The $1 \mathrm{~mm}$ thick sheets are represented in b) and c).

Mechanical measurements were done in a custom built mechanical testing machine. The tester consisted of a rigid frame; an electromagnetic linear motor provided the excitation, the stress on the sample was measured by a load cell mounted in the load path, and a capacitive sensor measured the displacement of the drive head. In all cases a sinusoidal drive was used. A temperature control chamber could be positioned around the sample to make measurements above room temperature.

For the scanning electron microscope (SEM) micrographs, slices about $1 \mathrm{~mm}$ thick where cut from the sample both normal and parallel to the direction of the field applied while curing. A variable pressure microscope was used, which allowed the samples to be observed without the need to coat them with a conductive layer.

Magnetic torque and the vibrating sample magnetometer measurements (VSM) were done on $6 \mathrm{~mm}$ diameter disks punched out of the sheets cured with the field normal to the plane, this direction correspond to the direction of the particle chains in the composite. A reference line was drawn on the surface of the sample, to be used as a reference direction on the sample to ensure that both stress and magnetic fields can be applied consistently to the sample.

For the torque measurements, the sample was placed on a transverse sample holder and a magnetic field was applied to the sample as it was rotated about the particle chain axis direction, always keeping the magnetic field in the plane of the sample. The sample was then removed from the sample holder and the sample was compressed in-plane along the direction of the reference line.

The stress on the sample was released and the sample was re-mounted on the sample holder and the torque measurement was repeated. This procedure was repeated several times, incrementally increasing the magnitude of the in-plane applied strain.

VSM measurements on the samples were first measured in the as-cast state with the magnetic field applied in the plane of the sample with the magnetic field parallel to the reference line. The sample was then removed from the sample holder and a compressive, in-plane stress was applied to the sample parallel to the reference line. The sample was the placed on the sample holder and was measured again.

X-ray diffraction experiments were done using the $\theta-2 \theta$ configuration, on sheets with both orientations of the curing field. The sample texture was analyzed on the sheets with the particlechain direction normal to the plane on which the X-rays are incident. Studies on the effect stress on the composite, were carried out on sheets cured with the magnetic field in the plane of the sample. The measuring X-ray beam again was incident on the plane of the sample. 
X-ray diffraction in NiMnGa alloys is less sensitive to low angle peaks that are the most representative of the variant's orientation. In that way neutron diffraction experiments are of much help and were also performed. The facilities available allow following in real time the diffraction of samples being simultaneously deformed. Such dynamic neutron diffraction measurements were done at the Institut Laue-Langevin in Grenoble, France. The D20 diffractometer was outfitted with a hydraulically driven Instron ${ }^{\circledR}$ testing machine such that the neutron beam would be perpendicular to the load path. The strain drive of the mechanical testing machine was used to trigger the acquisition of the neutron diffraction patterns, one hundred $20 \mathrm{~ms}$ diffraction patterns were taken over each deformation cycle and this was repeated over several hundreds of cycles, the corresponding diffraction patterns were added to achieve an acceptable statistic. The stress and strain on the sample were also collected.

\section{Results}

\section{Indirect observation of twin boundary motion}

The stress-strain response of both the matrix material and a $25 \%$ by volume composite of $\mathrm{Ni}-$ $\mathrm{Mn}-\mathrm{Ga}$ can be seen in Fig. 2 loaded under strain control up to $8 \%$. It can be seen that the area within the plot for the FSMA composite is far greater than the for matrix polymer for the same strain amplitude. The shapes of the traces should also be noted, the Polyurethane with no filler is a simple ellipse, the FSMA composite shows a more complex shape with three distinct slopes for the loop.
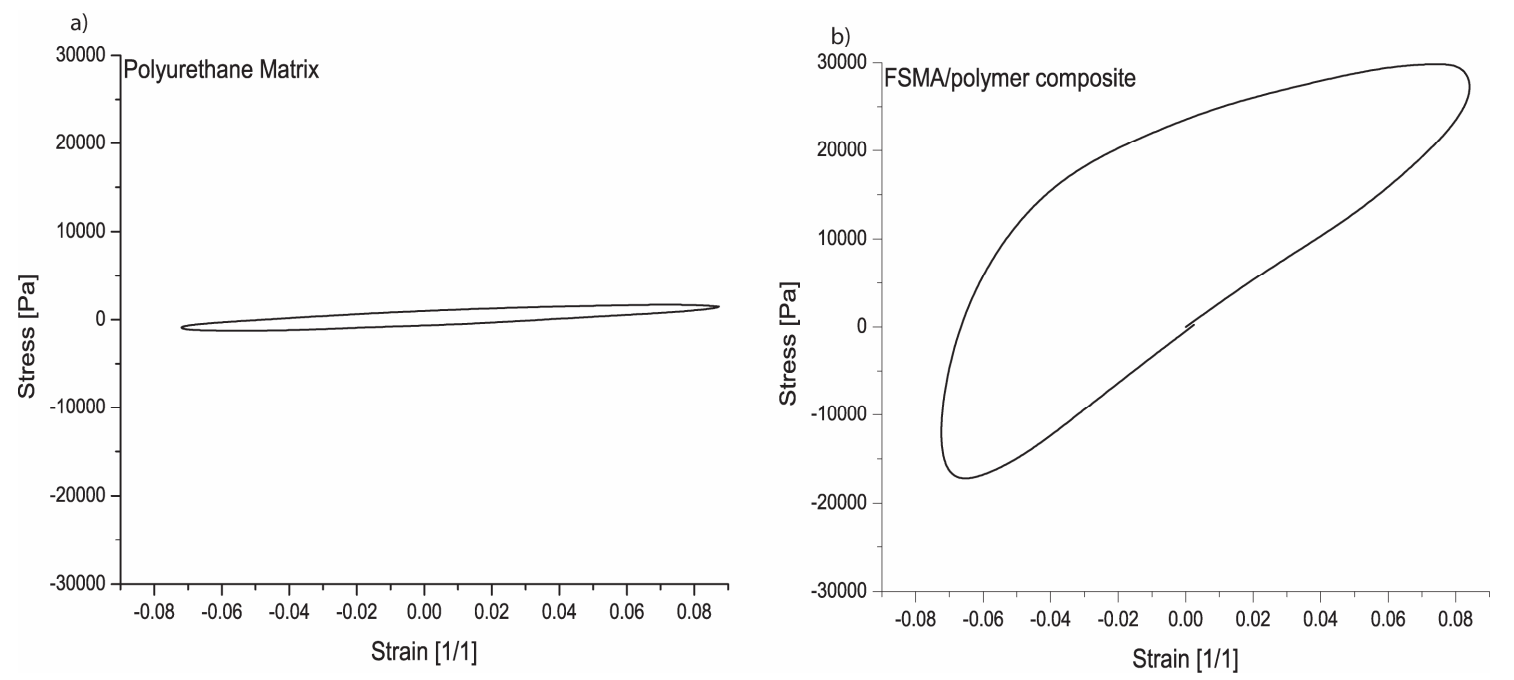

Figure 2: Stress vs. Strain plots for a) the polyurethane matrix used with no FSMA Filler and b) a 25 volume $\%$ FSMA in polyurethane composite, both measures at $0.5 \mathrm{~Hz}$. 
Fig 3 shows the maximum peak-to-peak magnetic torque measured as a function of the compressive strain applied to a Ni-Mn-Ga composite. As the compressive strain on the composite is increased, the peak-to-peak torque registered remains roughly constant at a low value. When a strain of $15 \%$ is reached, the peak-to-peak torque measured on the sample increases as the strain applied to the sample is increased. When a large compressive strain is applied normal to the plane of the sample, the peak-to-peak torque drops to the value originally measured, this is shown by the single circular marker in the graph.

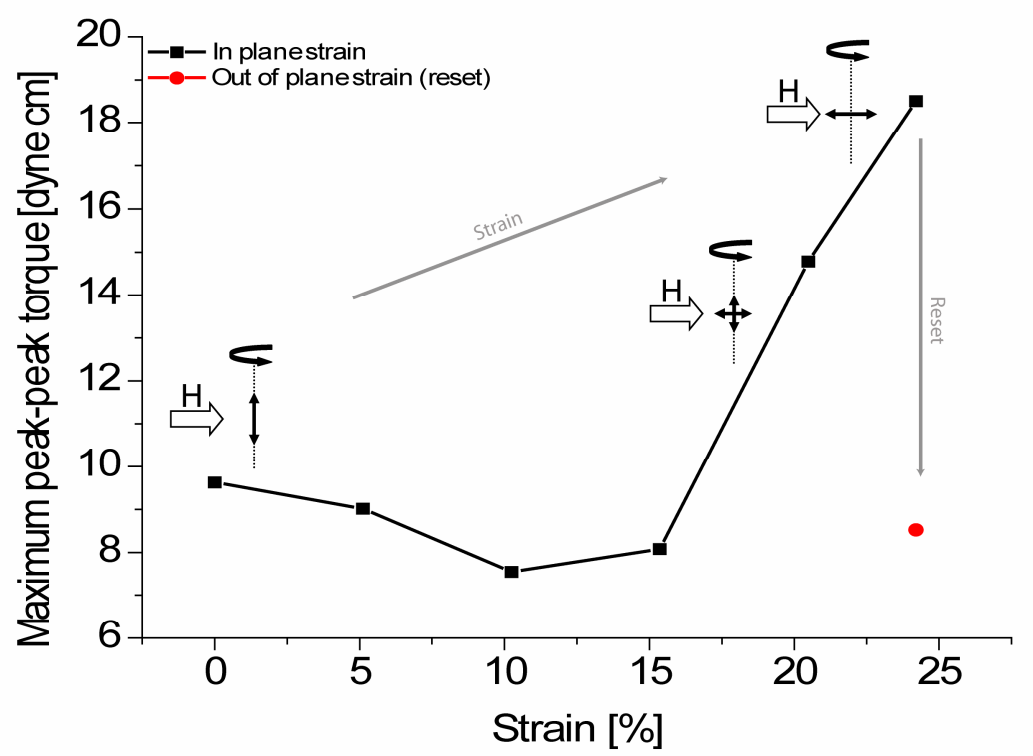

Figure 3: Peak magnetic torque as a function of in-plane compressive strain applied to a $25 \%$ by volume composite of $\mathrm{Ni}-\mathrm{Mn}-\mathrm{Ga}$ in polyurethane. The insets indicate the expected direction of the easy axis (represented by the double headed arrow) of the particles in the sample with respect to the axis of rotation (shown as a dashed line) and the direction of the magnetic field (bold arrow). 
Figure 4 shows three different VSM measurements done on the same sample maintaining the relative orientation of the reference line to the magnetic field for all measurements. The first trace, marked by squares, corresponds to the sample in the as cast state measured with an in-plane magnetic field. The second trace corresponds to the sample after a compressive strain was applied to the sample along the same direction as the magnetic field applied for the measurement. The third trace, marked by circles, corresponds to the composite sample heated to $100^{\circ} \mathrm{C}$ and allowed to cool down to room temperature under no applied stress or magnetic field. The trace for the sample heated up and cooled down falls in the middle of the other two traces shown in the graph.

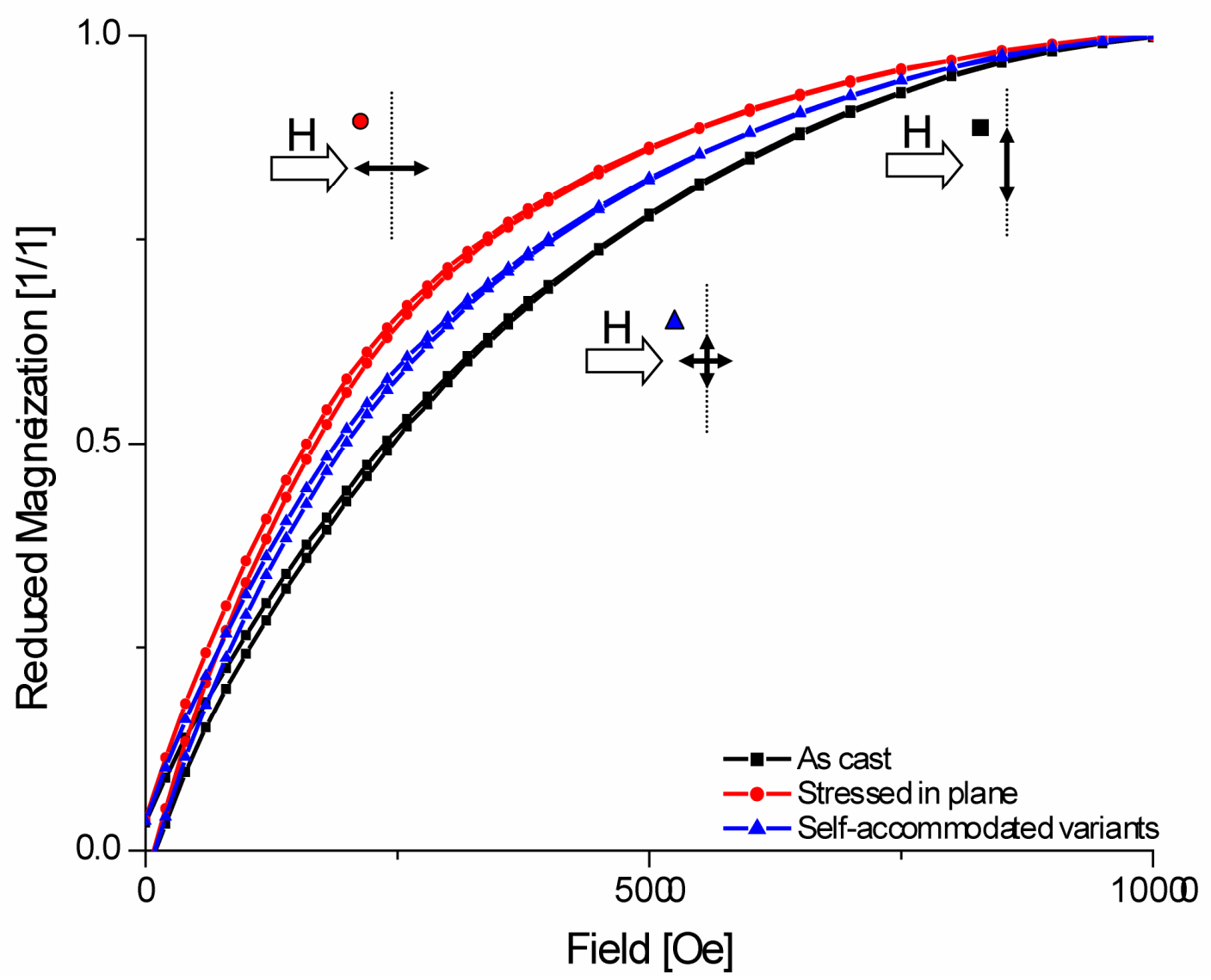

Figure 4 VSM measurement for a Ni-Mn-Ga composite, as-cast, after a compressive in-plane stress, and cooled to room temperature after heating it to $100^{\circ} \mathrm{C}$. 
The effect of the temperature on the ability of both the composite and the matrix material to dissipate energy can be seen in Fig. 5. The loss ratio is defined as the ratio of the amount of mechanical energy dissipated to the total mechanical energy used to drive the sample under cyclic loading. A clear difference in behavior can be seen for the composite and the matrix. In the case of the matrix the loss ratio initially increases as the temperature is increased, and then drops again to nearly its initial value. The FSMA composite on the other hand shows a loss that monotonically decreases with the increase in temperature.

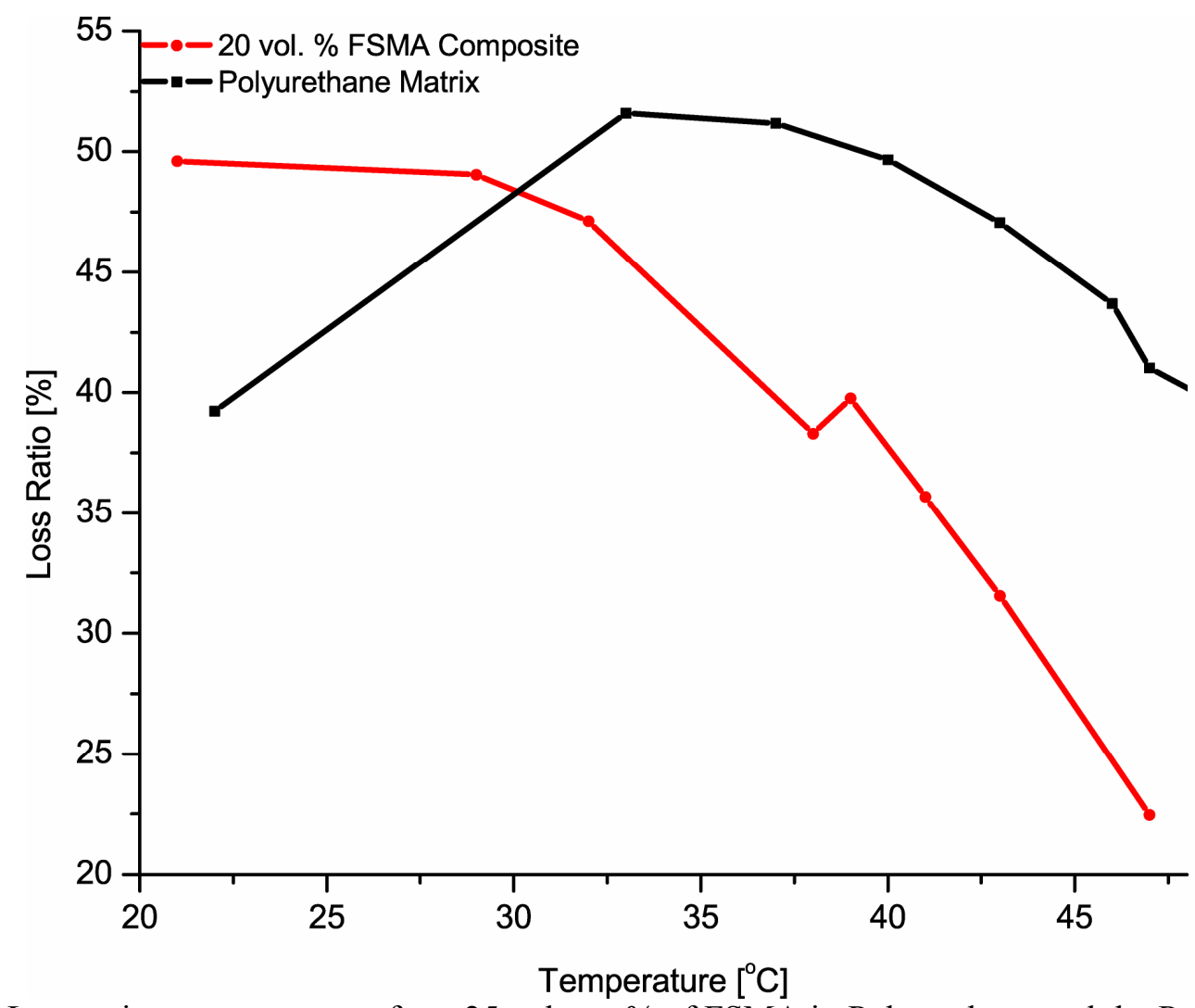

Figure 5: Loss ratio vs. temperature for a 25 volume \% of FSMA in Polyurethane and the Polyurethane with no filler. 
The effect of the stress amplitude on the loss ratio of both the composite and the matrix material is investigated at two different temperatures. The temperatures were chosen to be below and above the martensite transformation temperature of the FSMA filler $\left(\sim 45^{\circ} \mathrm{C}\right)$. As can be seen in Fig. 6 for both temperatures the loss ratio of the Polyurethane with no filler is mostly constant, while the loss ratio of the FSMA filled composite increases with increasing stress amplitude for the sample at room temperature, while the sample at $47^{\circ} \mathrm{C}$ shows no stress dependence of the loss ratio. It can be seen that at room temperature the composite has a higher loss ratio than the matrix material, but a $47^{\circ} \mathrm{C}$ the composite has a lower loss than the matrix material.
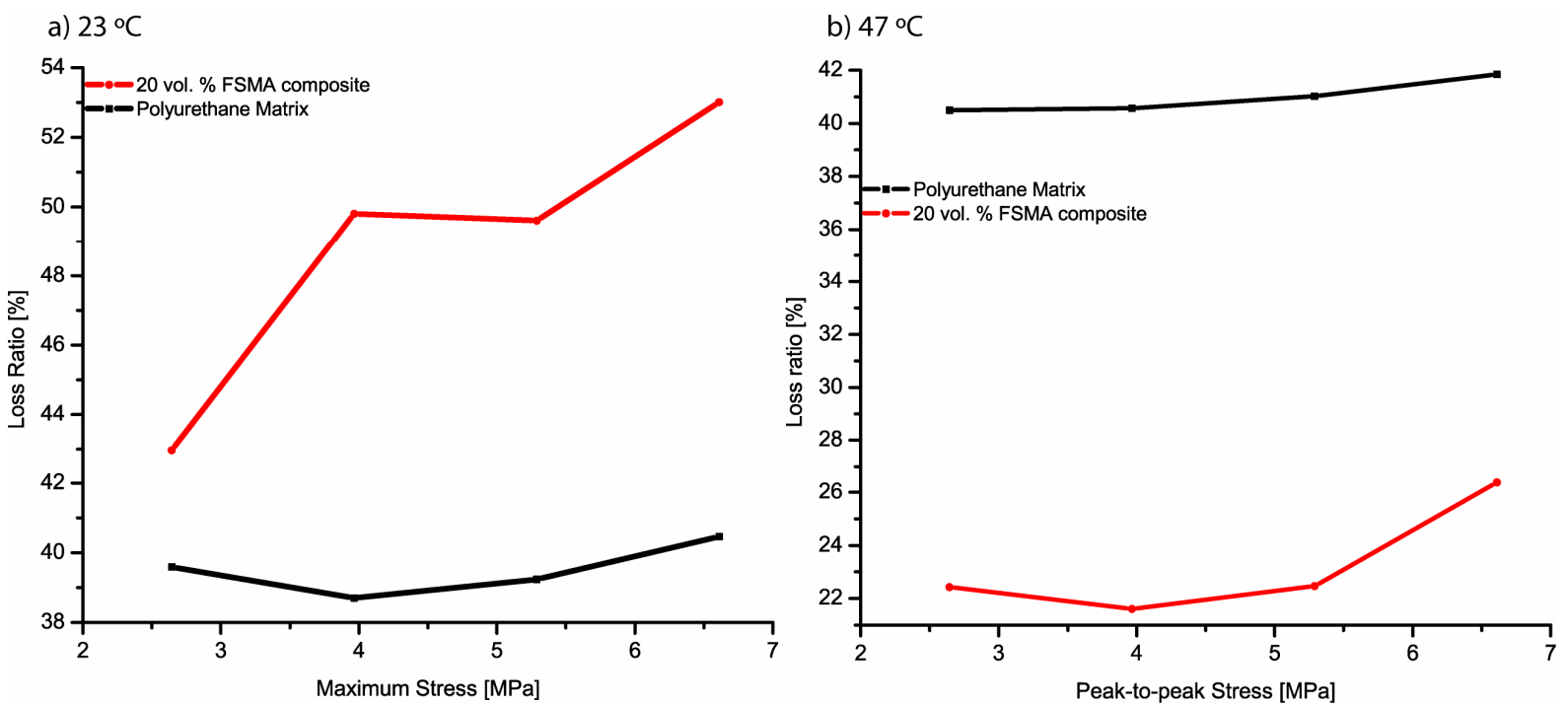

Figure 6: Loss ratio vs. stress amplitude for a 25 vol \% of FSMA in Polyurethane and the Polyurethane with no filler at a) $23^{\circ} \mathrm{C}$ and b) $47^{\circ} \mathrm{C}$. 


\section{Direct observation of twin boundary motion}

Fig. 7 shows backscatter SEM micrographs of a $\mathrm{Ni}-\mathrm{Mn}-\mathrm{Ga} /$ polyurethane composite. A micrograph of a plane that contains the direction of the magnetic field applied while curing the composite is shown in Fig. 7 a), clusters of particles forming elongated chain segments can be seen throughout the image. Fig. 7 b) shows a slice of the same composite, where the direction of magnetic field applied while curing is parallel to the image plane normal. Unlike figure 7 a) no elongated clusters of particles can be observed.

a)
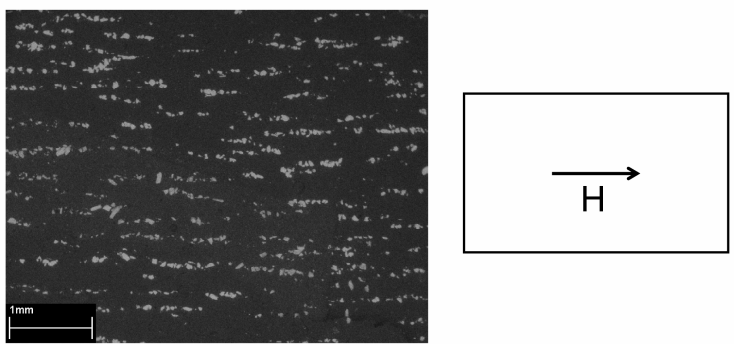

b)
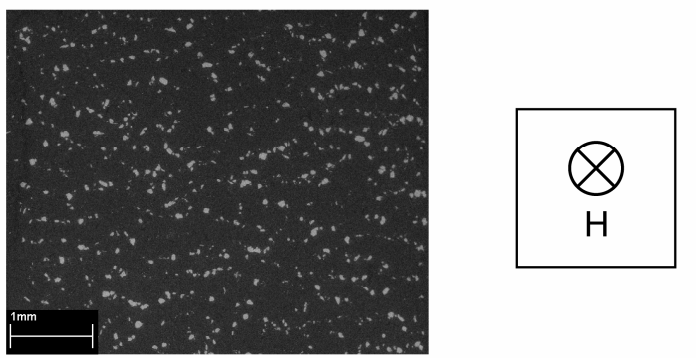

Figure 7: Backscatter SEM micrographs of a $10 \%$ by volume composite of Ni-Mn-Ga in Polyurethane cured under a magnetic field. a) shows a slice of the composite with the magnetic field direction in the plane of the image. b) shows a slice with the magnetic field normal to the image plane. 
In Fig. 8 a) the X-ray diffraction pattern for a $40 \%$ by volume composite of $\mathrm{Ni}-\mathrm{Mn}-\mathrm{Ga}$ in a polyurethane matrix, cured with no applied magnetic field is shown. Fig. $8 \mathrm{~b}$ ) shows the X-ray diffraction pattern for a $40 \%$ by volume composite of $\mathrm{Ni}-\mathrm{Mn}-\mathrm{Ga}$ in a polyurethane matrix, cured under a $4 \mathrm{kOe}$ magnetic field. For both graphs the intensities have been normalized to the amplitude of the (202) and (022) peak, which is the strongest peak. The intensity of the (004) peak shows a marked difference in relative intensities between the sample cured under a magnetic field and the sample cured with no applied field. The sample cured under a magnetic field shows a much larger relative intensity for the (004) peak than the sample cured with no applied magnetic field. As a comparison Fig. 9 shows the neutron diffraction patterns for Fig. 9 a) the filler material with no texture and b) for the composite cured under a magnetic field and no stress applied to it. Here too a variation in the relative intensities of the peaks can be seen for the textured and the random case.

a)
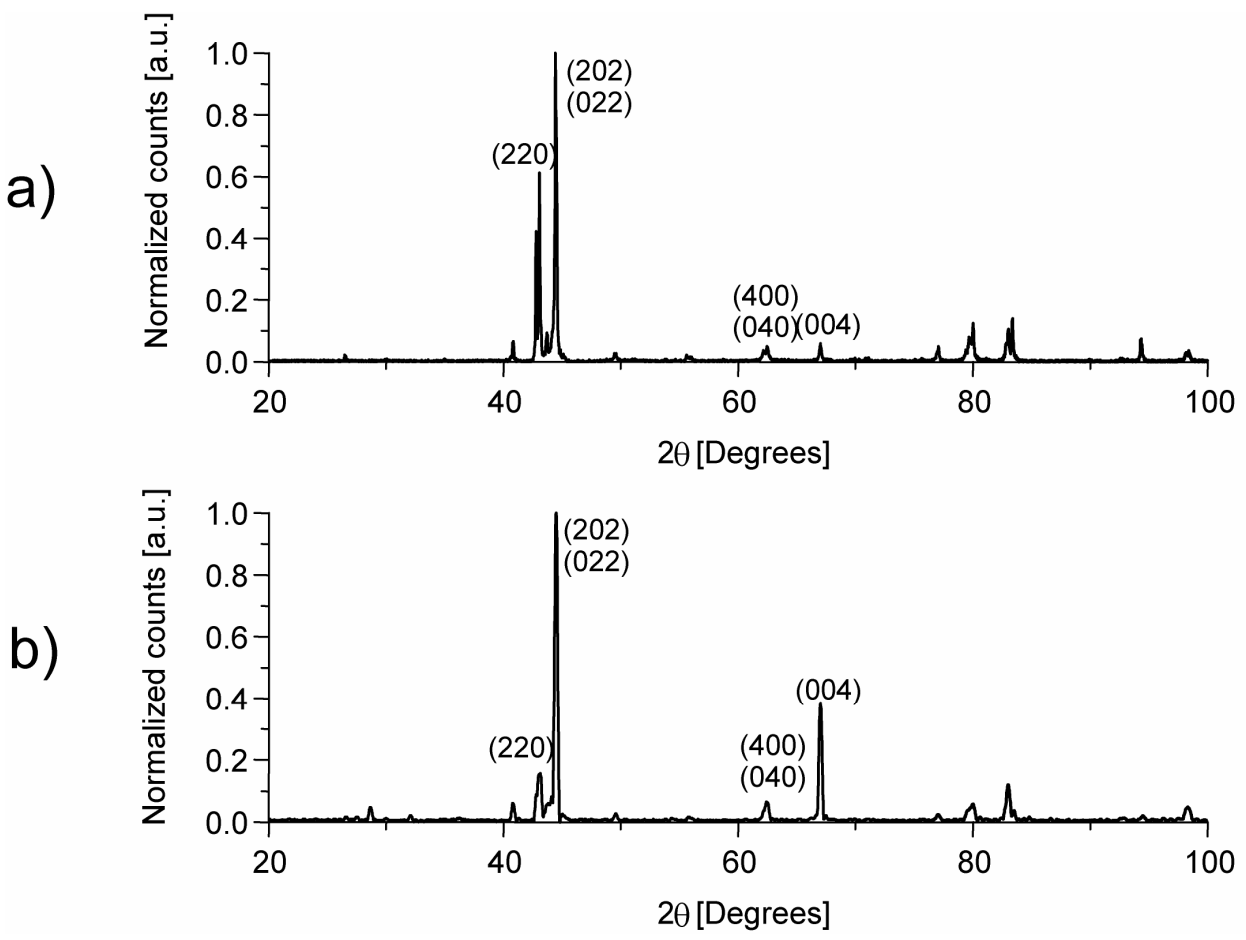

Figure 8: X-ray diffraction pattern for a) a composite cured with no applied magnetic field and b) for a composite cured under a magnetic field, $\lambda=1.54 \AA$. 




Figure 9: Neutron diffraction pattern for a) the FSMA filler with no texture and $b$ ) for a composite cured under a magnetic field. The neutron beam in this case incided normal to the particle chains $\lambda=1.87 \AA$.

More peaks can be seen when using neutron instead of x-rays because the difference in scattering factors for the $\mathrm{Mn}$ and the $\mathrm{Ga}$ are greater when neutrons are used.

Fig. 10 shows the X-ray diffraction pattern for a sheet of $\mathrm{Ni}-\mathrm{Mn}-\mathrm{Ga} /$ polymer composite. Fig. 10 a) shows the diffraction pattern for the composite as cast, the intensities have been normalized to the intensity of the (400) and (040) peak. The dashed line marks the relative height of the (004) peak. The initial intensity of the (004) is approximately $50 \%$ of the (400) and (040) peak. Fig. $10 \mathrm{~b}$ ) shows the diffraction pattern for the same sheet of composite after a large compressive stress applied normal to the plane where the X-rays are incident. The intensity of the (004) peak relative to the (400) and (040) peak has increased to approximately $70 \%$. 
(a)

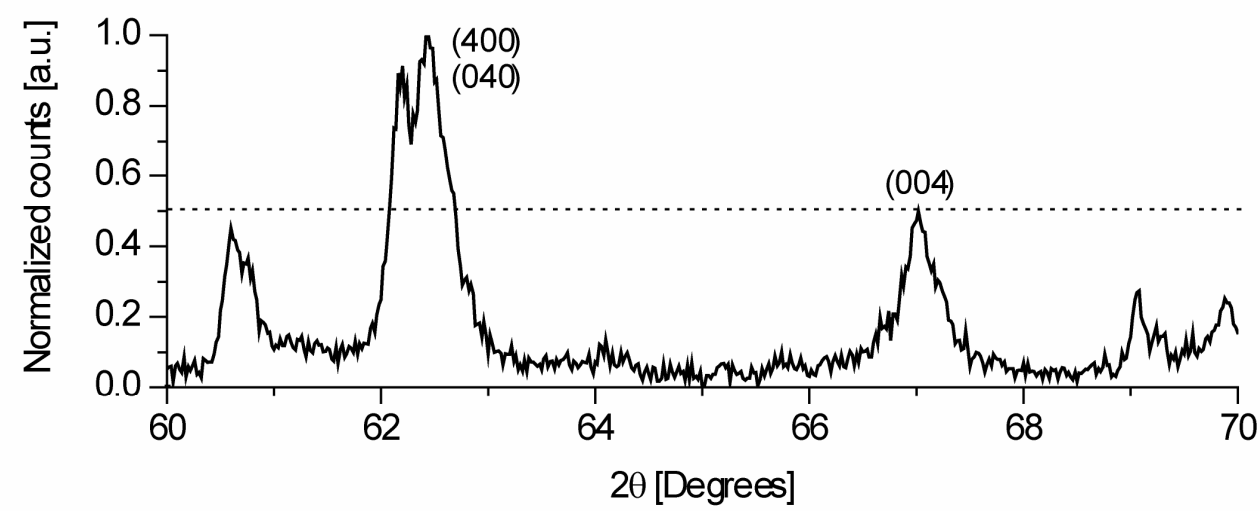

(b)

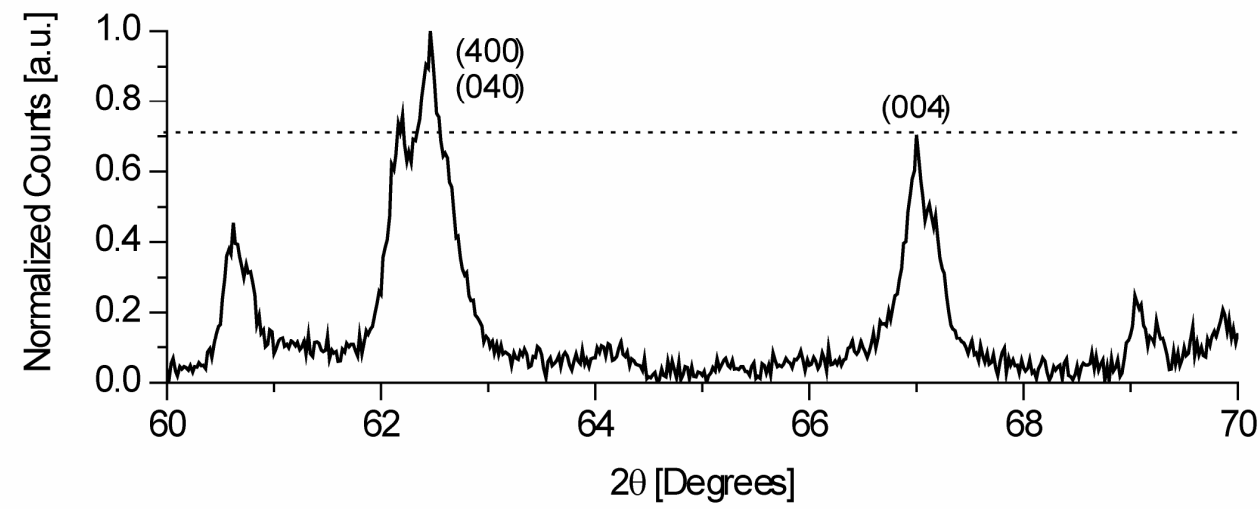

Figure 10: X-ray diffraction pattern for a composite of $\mathrm{Ni}-\mathrm{Mn}-\mathrm{Ga}$ a) in the as cast state, b) after it was subjected to a compressive stress along the direction of the sheet normal. $\lambda=1.54 \AA$

Similar to the results in Fig. 10, in Fig. 11 the intensity of the (020) and the (112) martensite peaks can be observed as a function of time, while the sample was subjected to a $0.5 \mathrm{~Hz}$ strain, while under the neutron beam. The diffraction pattern showed that these peaks (not seen under X-rays) corresponded to an orthorhombic martensite. It can be seen that the intensity of one increases as that of the other decreases. From that figure the area ratios for the (020) to the (112) peaks could be obtained by fitting Lorentzians to the diffraction peaks at each of the 100 slices. The ratio of the areas at each slice was then taken and plotted as a function of time. As can be seen in Fig. 12 the ratio of the peaks follows a sinusoidal that roughly coincides with the drive strain, but with the phase reversed. The stress also shows additional features and is not a clean sine wave. 


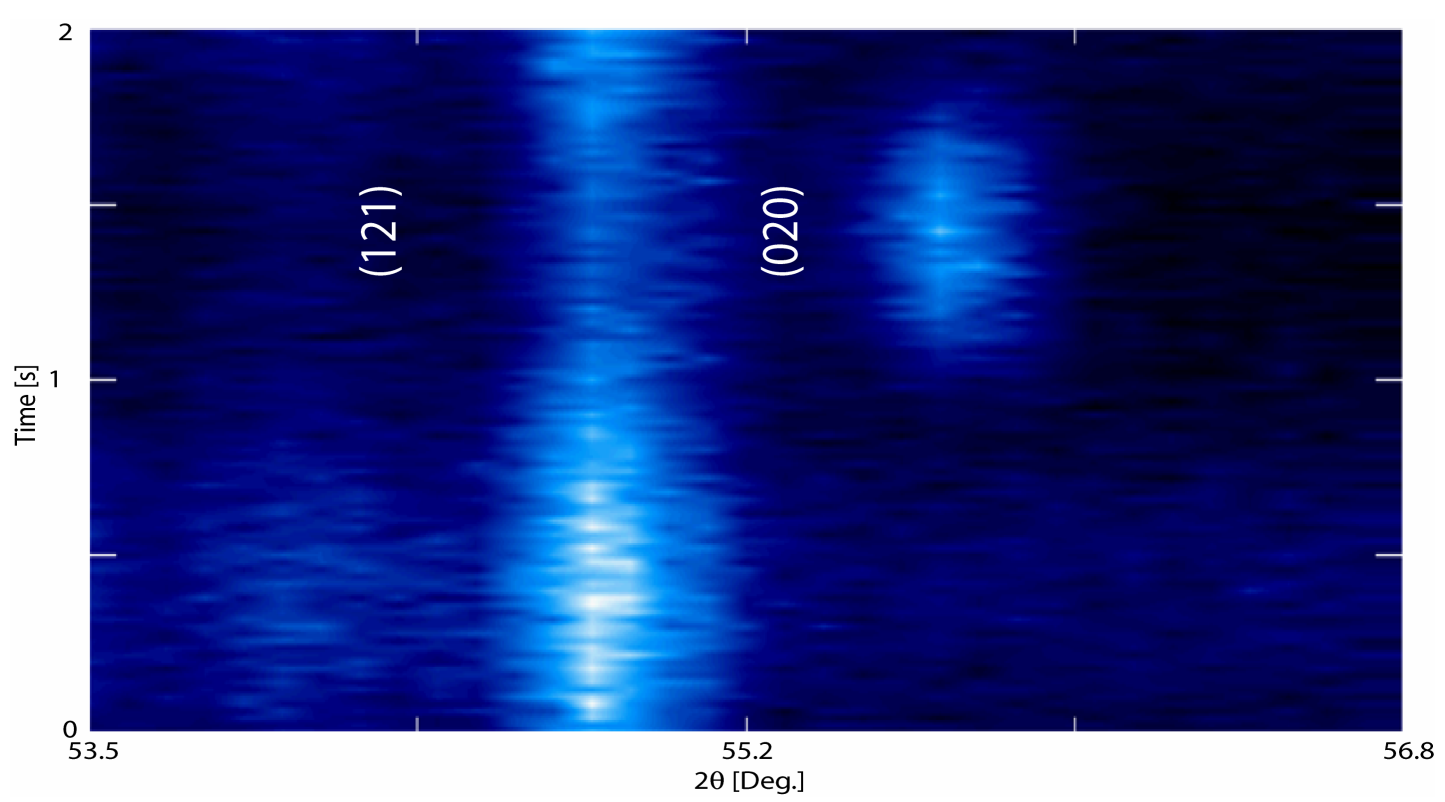

Figure 11: Intensity map for the (020) and the (112) peaks as a function of time for a composite subjected to a $0.5 \mathrm{~Hz}$ sinusoidal strain. , $\lambda=1.87 \AA$

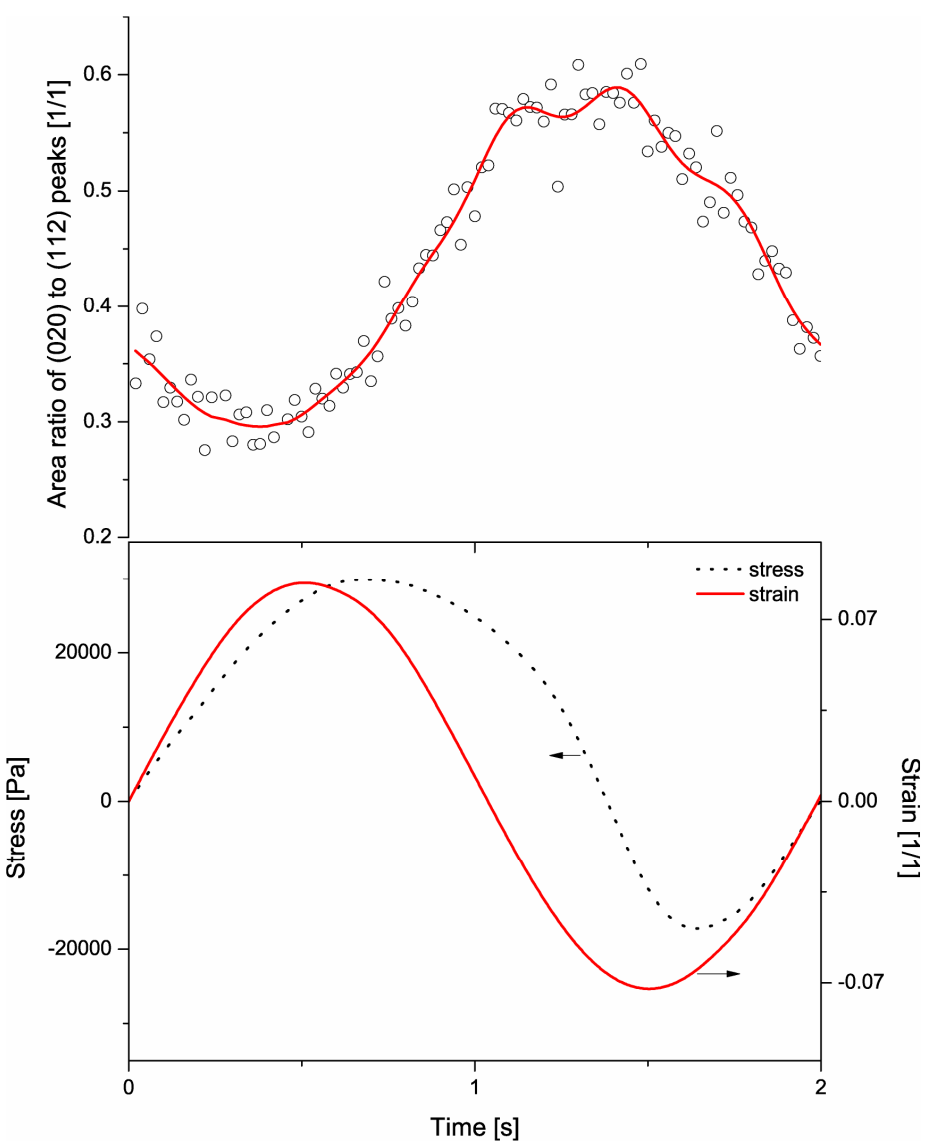

Figure 12: Top: Area ratio of the (020) peak to the (112) peak as a function of time, open circles are the measured data and the solid trace corresponds to the smoothing of the data using an FFT algorithm. Bottom: Stress and strain as a function of time collected during the neutron diffraction experiment.

\section{Discussion}

Fig. 7 shows that applying a magnetic field to the composite as the polymer cures causes the particles to align in chains along the direction of the magnetic field. This however does not necessarily guarantee that the particles will be properly oriented in the composite. For the particles 
to be properly oriented, the composite should show a strong 001 texture along its long axis. This texture allows for stresses applied along, or normal, to the long axis to be resolved in the particles as a shear along the $\{110\}$ planes. A resolved shear along the $\{110\}$ planes is needed to move the twin boundaries in Ni-Mn-Ga. Fig. 8 shows that the needed texture is present in the composites. This can be seen by the marked increase in the relative intensity of the (004) peak for the composite cured under a magnetic field, as can be seen in Figure $8 \mathrm{~b}$ ). The particle alignment and orientation in the composite fulfill the initial requirements for a composite to be mechanically active.

Fig. 3 shows that when a gradual in-plane strain is applied to the composites a threshold behavior, reminiscent of the behavior of a single crystal of $\mathrm{Ni}-\mathrm{Mn}-\mathrm{Ga}$ under a compressive load is observed. Only magnetic moments in the plane with a normal parallel to the axis of rotation will cause a torque that can be registered by the torque magnetometer. As can be seen in the insets in Fig. 3, the composite as cast ( $0 \%$ strain) has a large fraction of the easy axes in the particles that are parallel to the axis of rotation. As the in-plane strain on the composite is increased, the variants with easy axes normal to the axis of rotation grow, and bring the magnetic moments into the plane where they can cause a torque that can be registered by the magnetometer. The torque increases gradually as the particles go from single variants out of plane, to a multi-variant state and to a single variant with the easy-axis rotated $90^{\circ}$ with respect to the initial configuration. Since the strain was applied to the composites and then released before the sample was placed in the magnetometer, the effect observed is not an elastic process, but rather a plastic one. This behavior is indicative of twin boundary motion, but is not a direct confirmation of the motion of twin boundaries. Fig. 10 on the other hand directly confirms that a stress applied to the composite causes the motion of twin boundaries. The change in the relative intensities of the (004) peaks cannot be caused by plastic deformation. The neutron diffraction data goes one step beyond the X-ray data, showing in real time the stress-induced variant change in the composite. The data is not clean enough to shed light on the yield stress distribution for the twins in the particles, but it shows clearly that there is a relation between the strain applied to the composite and the variant distribution in the particles.

For the particles to orient properly in the composite they have to be single variant or mostly single variant. The particles used in the composite were first placed loosely in a container and placed in a magnetic field and a stress was applied in the same direction as the magnetic field.

This process was repeated several times to induce one dominant variant in the particles. Fig. 4 shows the magnetization loop (square markers) for the composite in the as cast state, as can be seen in the inset, the easy axes of the particles are perpendicular to the magnetic field used in the measurement. The shape of this loop does not change if a compressive stress is applied normal to the plane of the sample. This indicates that all the mobile twin boundaries have moved and no more easy axes can be rotated into the direction of the applied stress. When an in plane stress is applied to the sample, the shape of the magnetization loops changes, and it more closely resembles an easyaxis magnetization loop (circular markers), and applying larger stresses in that direction no longer changes the shape of the loops. This two traces mark the two extremes of how far the moments can be moved in the composite. The loop with the triangle markers corresponds to the sample after heating it up above its austenite finish temperature and then allowing it to cool down below its martensite finish temperature. This trace falls exactly between the two that mark the extremes, indicating that the particles formed self accommodated martensite with roughly equal volume fractions of the different variants. A composite with particles that have multiple variants would fall between the two other traces. If the particles had been multi-variant when the composites were made, the as-cast magnetization loop would have followed the self accommodated loop very closely. Since the loop for the as cast composite shows a harder character than the self accommodated loop, it follows that the variant distribution in the particles used was different from the self accommodated configuration. 


\section{Conclusions}

Curing the polyurethane/FSMA slurry under a field yielded pseudo 3:1 composites that have the appropriate crystallographic texture for the twin boundaries in the particles within the composites to respond to a stress applied to them along the direction of the magnetic field used while curing the composites. The mobility of the twin boundaries was determined indirectly by the torque magnetometry measurements on the samples that show that an incremental increase in the torque can be observed as the magnitude of the applied strain is increased, after a threshold value is reached. X-ray diffraction measurements before and after stressing the composite show direct evidence that the changes observed in the torque measurements are the result of a change in the variant distribution in the particles. The neutron diffraction data fully confirms the static X-ray diffraction data and helps show that the additional loss observed in the stress-strain loops is due to the motion of twin boundaries. Finally, the VSM measurements showed that the particles used to make the composites were successfully preconditioned to obtain mostly a single variant state. This is key to obtaining the initial texture in the composites. All the evidence presented consistently shows that applying a large enough stress to the composites can cause the twin boundaries to move in the filler particles, as is shown indirectly by magnetic measurements and directly by diffraction experiments. It is also shown that the additional loss observed in the composites disappears as the temperature of the composite is increased above the austenite transformation temperature, and that the additional depends on the amplitude of the applied load. Put together all the evidence shows with great certainty that the FSMA composites owe their additional loss mostly to the motion of twin boundaries.

\section{Acknowledgements}

The authors would like to acknowledge the Institute Laue-Langevin for the use of the neutron diffraction facilities. The US Navy's Office of Naval Research for their support through MURI grant No. N0014-01-0758. The Basque Government's Department of Industry for their support through the ACTIMAT program. J. F. would like to acknowledge the support of the European Commission through the Marie Curie IIF program under FP6 for his support.

\section{References}

[1] S. J. Murray, M. A. Marioni, S. M. Allen, R. C. O’Handley and T. A. Lograsso: Appl. Phys. Lett. Vol.77 (2000), p.886.

[2] A. Sozinov, A. A. Likhachev, N. Lanska, K. Ullakko and V. K. Lindroos: In Smart Structures and Materials. Proceedings of SPIE Vol. 4699 (2002), p.195.

[3] Jan Van Humbeeck: Shape memory alloys: A material and a technology. Advanced Engineering Materials Vol. 3(11) (2001), p.837.

[4] C. P. Henry, J. Feuchtwanger, D. Bono, M. A. Marioni, P. G. Tello, M. Richard, S. M. Allen, and R. C. O'Handley: Ac performance and modelling of ferromagnetic shape memory actuators. In Smart Structures and Materials Proceedings of SPIE Vol. 4333 (2001), p.151.

[5] M. A. Marioni, R. C. O'Handley and S. M. Allen: Pulsed magnetic field-induced actuation of Ni-Mn-Ga single crystals. Appl. Phys. Lett. Vol.83 (2003), p.3966.

[6] C. M. Harris: ed. Harris’ Shock and Vibration Handbook. McGraw-Hill, 5th edition, 2002. 
[7] Y.C. Wang, M. Ludwigson and R.S. Lakes: Deformation of extreme viscoelastic metals andcomposites. Materials Science and Engineering $A$ Vol. 370 (2004), p.41.

[8] J. P. Teter, M. Wunfogle, A. E. Clark and K. Mahoney: Anisotropic perpendicular axis magnetostriction in twinned $\mathrm{Tb}_{x} \mathrm{Dy}_{1-x} \mathrm{Fe}_{1.95}$. Journal of Applied Physics Vol. 67 (1990), p.5004.

[9] G. P. McKnight and G. P. Carman: Energy absorption and damping in magnetostrictive composites. Mat. Res. Soc. Symp Proc. Vol.604 (2000), p.267.

[10] C. Rodriguez, A. Barrio, I. Orue, J.L. Vilas, L.M. Leon, J. M. Barandiaran, M.L. FdezGubieda Ruiz: High magnetostriction polymer-bonded Terfenol-D composites, Sensors and Actuators A: Physical Vol.142 (2008), p.538.

[11] N. Scheerbaum, D. Hinz, O. Gutfleisch, K.-H. Muller, and L. Schultz: Acta Mat., Vol. 55 ( 2007), p.2707.

[12] D. Hinz, N. Scheerbaum, O. Gutfleisch, K.-H. Muller and L. Schultz: JMMM Vol.310 (2007) p.2785.

[13] Eric Gans, Chris Henry and Gregory P. Carman: High-energy absorption in bulk ferromagnetic shape memory alloys $\left(\mathrm{Ni}_{50} \mathrm{Mn}_{29} \mathrm{Ga}_{21}\right)$. In Dimitris C. Lagoudas, editor, Proceedings of SPIE Smart Structures and Materials 2004: Active Materials: Behavior and Mechanics Vol. 5387 (2004), p.177.

[14] J. Feuchtwanger, S. Michael, J. K. Huang, D. Bono, R. C. O’Handley, S. M. Allen, K. Jenkins, J. Goldie and A. Berkowitz: Energy absorption in Ni-Mn-Ga polymer composites. J. Appl. Phys. Vol. 93 (2003), p.319.

[15] J. Feuchtwanger, K. Griffin, J. K. Huang, R. C. O’Handley, S. M. Allen and D. Bono: Vibration damping in Ni-Mn-Ga-polymer composites. In Smart Structures and Materials. Proceedings of SPIE SS/NDA, 2003.

[16] J. Feuchtwanger, K. Griffin, J. K. Huang, D. Bono, R. C. O'Handley and S. M. Allen: Mechanical energy absorption in Ni-Mn-Ga polymer composites. J. Magn. Magn. Mat., Vol. 272276(2004), p.2038.

[17] J. Feuchtwanger, M. L. Richard, Y. J. Tang, A. E. Berkowitz, R. C. O'Handley and S. M. Allen: Large energy absorption in Ni-Mn-Ga/polymer composites. J. Appl. Phys. Vol.97 (2005), $10 \mathrm{M} 329$.

[18] J. Feuchtwanger, P. Lázpita, N. Vidal, J. M. Barandiaran, J. Gutiérrez, T. Hansen, M. Peel, C. Mondelli, R. C. O'Handley and S. M. Allen: Rearrangement of twin variants in ferromagnetic shape memory alloy-polyurethane composites studied by stroboscopic neutron diffraction. J. Phys.:

Condens. Matter Vol. 20 (2008)104247.

[19] J. Carrey, H. B. Radousky and A. E. Berkowitz: Spark-eroded particles: Influence of Processing parameters. Journal of Applied Physics Vol. 95 ( 2004), p.823.

[20] A.E. Berkowitz, M.F. Hansen, F.T. Parker, K.S. Vecchio, F.E. Spada, E.J. Lavernia and R. Rodriguez: Amorphous soft magnetic particles produced by spark erosion. Journal of Magnetism and Magnetic Materials Vol. 254 (2003), pp. 1-6 . 